\title{
Microscale resolution fracture toughness profiling at the zirconia- porcelain interface in dental prostheses
}

\author{
Alexander J. G. Lunt*a, Gaurav Mohanty ${ }^{\mathrm{b}}$, Tee K. Neo ${ }^{\mathrm{c}}$, Johann Michler ${ }^{\mathrm{b}}$ \& Alexander M. \\ Korsunsky ${ }^{\mathrm{a}}$ \\ ${ }^{a}$ The Multi-Beam Laboratory for Engineering Microscopy, Department of Engineering Science, \\ University of Oxford, Parks Road, Oxford, Oxfordshire, OX1 3PJ, United Kingdom. \\ ${ }^{\mathrm{b}}$ Empa Materials Science \& Technology, Thun, Feuerwerkerstrasse 39, CH-3602, Switzerland. \\ ${ }^{\mathrm{c}}$ Specialist Dental Group, Mount Elizabeth Orchard, 3 Mount Elizabeth, \#08-03/08-08/08-10, \\ Singapore 228510.
}

\begin{abstract}
The high failure rate of the Yttria Partially Stabilized Zirconia (YPSZ)-porcelain interface in dental prostheses is influenced by the micro-scale mechanical property variation in this region. To improve the understanding of this behavior, micro-scale fracture toughness profiling by nanoindentation micropillar splitting is reported for the first time. Sixty $5 \mu \mathrm{m}$ diameter micropillars were machined within the first $100 \mu \mathrm{m}$ of the interface. Berkovich nanoindentation provided estimates of the bulk fracture toughness of YPSZ and porcelain that matched the literature values closely. However, the large included tip angle prevented precise alignment of indenter with the pillar center. Cube corner indentation was performed on the remainder of the pillars and calibration between nanoindentation using different tip shapes was used to determine the associated conversion factors. YPSZ micropillars failed by gradual crack propagation and bulk values persisted to within $15 \mu \mathrm{m}$ from the interface, beyond which scatter increased and a $10 \%$ increase in fracture toughness was observed that may be associated with grain size variation at this location. Micropillars straddling the interface displayed preferential fracture within porcelain parallel to the interface at a location where nano-voiding has previously been observed and reported. Pure porcelain micropillars exhibited highly brittle failure and a large reduction of fracture toughness (by up to $\sim 90 \%$ ) within the first $50 \mu \mathrm{m}$ of the interface. These new insights constitute a major advance in understanding the structure-property relationship of this important bi-material interface at the micro-scale, and will improve micromechanical modelling needed to optimize current manufacturing routes and reduce failure.
\end{abstract}

Keywords: Fracture toughness, spatially resolved, yttria partially stabilized zirconia, porcelain, nanoindentation, micropillar splitting, microscale, dental prostheses

\section{INTRODUCTION}

Yttria Partially Stabilized Zirconia (YPSZ) is a high strength, high toughness ceramic which in recent years has found increasing use in the manufacture of dental prostheses [1]. The origins of the toughening behavior of this material lie in the tetragonal to monoclinic YPSZ phase transformation which may occur in the vicinity of cracks [2], free surfaces and interfaces. In dental applications YPSZ copings are veneered with porcelain in order to produce an aesthetically pleasing finish and to reduce the hardness of the outer surface, thereby reducing wear on existing opposing teeth [3]. However, this approach leads to the primary failure mode of YPSZ prostheses: near interface chipping of the porcelain veneer [4]. The origins of this failure are poorly understood but are believed to be linked to the mechanical property variation, residual stresses and porcelain creep induced in the near-interface region during prostheses manufacture [5-9].

Microanalysis and compositional mapping of the YPSZ-porcelain interface has been performed using a broad range of experimental techniques including Scanning Electron Microscopy (SEM) [10, 11], Energy Dispersive Spectroscopy (EDS) [11, 12], Transmission Electron Microscopy (TEM) [11, 13] and micro-focus X-ray diffraction [10, 14]. These studies have demonstrated that the microstructural variation at the YPSZ-porcelain interface is limited to within the first few hundred microns of the interface, and that the behavior is influenced by both nano-voiding and nano-grain features. However, these studies do not provide insight into the mechanical properties of most significance for prosthesis integrity: stiffness, hardness (yield strength) and toughness. Recently, microscale spatially resolved studies of Young's modulus and yield strength variation across the YPSZ-porcelain interface region have recently been published $[11,15,16]$. The

SPIE Micro+Nano Materials, Devices, and Systems, edited by Benjamin J. Eggleton, Stefano Palomba Proc. of SPIE Vol. 9668, 96685S · @ 2015 SPIE · CCC code: 0277-786X/15/\$18 · doi: 10.1117/12.2199217 
analysis of toughness variation at very short length scales has remained an insurmountable challenge, despite the fact that material resistance to crack initiation and propagation, is critical for understanding the origins of the brittle failure observed at the YPSZ-porcelain interface. Quantification of the fracture toughness of both bulk YPSZ [17, 18] and bulk porcelain $[19,20]$ has been widely reported. However, no microscale spatially resolved analysis has been performed to determine the variation in the vicinity of the interface where failure is known to originate [21].

One of the most well-established and widely used procedures for microscale fracture toughness experimental determination is based on the measurement of the length of cracks induced by nanoindentation. It was first developed by Evans et al [22] in 1976. In recent years this approach has been refined to improve the resolution and precision of the technique, however it remains highly sensitive to the shape of the crack, the exact shape of the indenter tip (which may vary due to manufacturing imperfections and variation in use), and substrate mechanical properties [23]. Further, the impact of residual stress is known to influence crack formation in this configuration. This has been demonstrated in a recent study by Sebastiani et al. [15] who used nanoindentation to quantify the average values of fracture toughness within four regions across a YPSZ-porcelain cross section, in which one average near-interface value was reported. Since the magnitude of residual stress at the interface is known to be large and rapidly varying [16], de-convolution of this effect cannot be reliably performed for nanoindentation results at the micrometer resolution required.

Recent improvements in nanoindentation systems and the increased availability of SEM-Focused Ion Beam (FIB) microscopes have facilitated the development of several new high resolution fracture toughness analysis techniques. A particular highly successful class of these methods is based around FIB milling and loading of notched micro-cantilevers [24-26]. Although these approaches benefit from high precision of crack positioning, there is currently disagreement in the literature over the sensitivity of the approach to the friction at the interface between the indenter surface (diamond) and the substrate, and the influence of ion beam damage at notch roots. The large FIB milling times required for each measurement point also limits the applicability of this approach for spatially resolved study.

An alternative microscale fracture toughness approach based on Berkovich nanoindentation and splitting cracking of micropillars has recently been proposed by Sebastiani et al. [23, 27]. This approach benefits from a well-defined microscale gauge volume and fracture is known to originate from a location unaffected by FIB milling. Furthermore, complete relief of residual stress takes place within the examined region prior to indentation, minimizing the impact of this effect on the result. Additionally, the method can be implemented in a relatively short time frame, opening the possibilities of fracture toughness profiling and mapping. These benefits make it highly suitable for spatially resolved fracture toughness analysis across the YPSZ-porcelain interface.

\section{METHODOLOGY}

\subsection{Sample preparation}

An incisal YPSZ-porcelain dental prosthesis was manufactured at DE Dental Lab Pte Ltd, Singapore. The assembly process employed was identical to that used in commercial samples: a YPSZ prostheses coping was produced by shaping lightly pre-sintered Zenotec Zr Bridge [28] into the form required. This was then sintered at $1600^{\circ} \mathrm{C}$ to produce a fully dense component. Multiple layers of IPS e.max Ceram [29] were then applied to the surface of the coping as aqueous slurry and fired at temperature around $650^{\circ} \mathrm{C}$ in order to produce a smooth porcelain surface with a realistic and aesthetically pleasing finish.

In order to gain access to the interface, the completed prosthesis was mounted in epoxy resin and a $2 \mathrm{~mm}$-thick cross sectional slice was obtained using Buehler Isomet Diamond Saw. An incremental grinding and polishing process was then used to remove any material influenced by the cutting process and to minimize the magnitude of residual stresses induced by sample preparation. The sample was then mounted on an SEM stub using silver paint to provide an effective conduction path and minimize charging during FIB milling and SEM imaging.

\subsection{Micropillar FIB machining}

FIB milling was used to generate an array of micropillars in the shape of a cross straddling the interface as shown in Figure 1a. This pattern was selected to maximize the number of pillars within the first $15 \mu \mathrm{m}$ of the interface in order to give improved statistics in the location where most variation was expected. FIB milling was performed on the TESCAN Lyra 3 FIB-SEM at the Multi-Beam Laboratory for Engineering Microscopy (MBLEM), Oxford, UK using an automated script for pillar milling, and a milling current of $1.25 \mathrm{nA}$. Nominal pillar diameter and depths of $5 \mu \mathrm{m}$ were selected in order to provide the required resolution while simultaneously minimizing the impact of ion beam damage on 
the fracture toughness evaluation process. This size was also consistent with the successful experiments previously performed by Sebastiani et al [27]. A trench width of $3 \mu \mathrm{m}$ was selected as a compromise between maximizing the spatial resolution of the data collected and ensuring that there was no contact or interaction between the indenter and the surrounding material. In order to aid in aligning the indenter on the center of each pillar, a $25 \mathrm{~nm}$ deep target cross was milled into the surface, as shown in Figure 1b. High resolution imaging was performed on each pillar in using SEM stage tilt angles of $0^{\circ}$ and $35^{\circ}$ in order to measure the radius $(R)$ and height of each pillar using the technique previously outlined by Lunt et al. [11]. Precise measurement of the distance from the interface to the center of each pillar was also performed during this process.
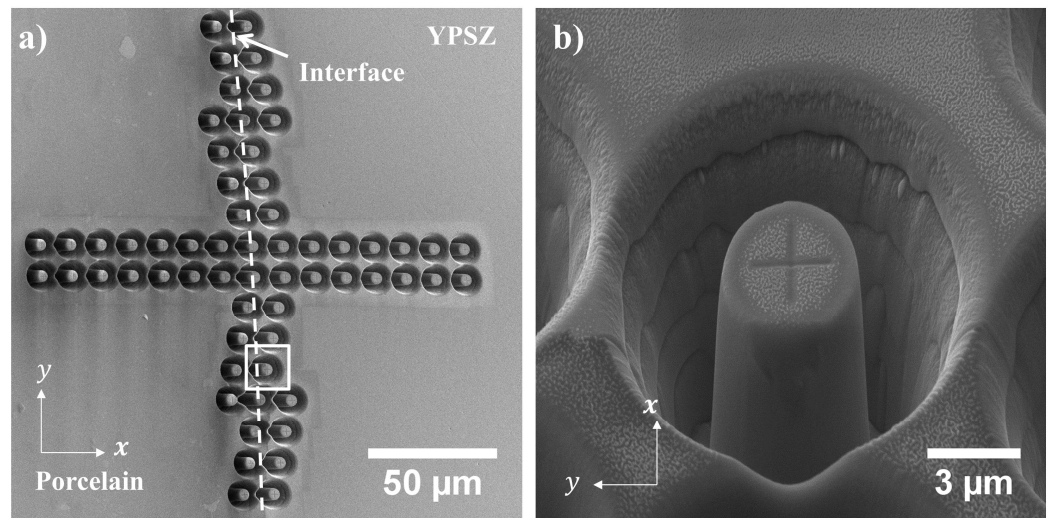

Figure 1. a) Positions of FIB milled micropillars across the YPSZ-Porcelain interface. The number of pillars near to the interface has been chosen to be large in order to gain improved insight into the statistics of this region. b) A high magnification of one of the pillars (the location of which is shown in Figure 1a showing the indentation alignment cross on the center of the pillar). Both images were captured at a SEM tilt of $55^{\circ}$ using tilt correction in the $x$ direction. Figure 1a has been rotated to be orientationally consistent with the other figures presented in this study.

\subsection{Micropillar indentation splitting}

Micropillar indentation splitting was performed using an Alemnis SEM Indenter inside the TESCAN Lyra 3 FIB-SEM at Empa, Thun, Switzerland. Prior to the start of the experiment, compliance calibration was performed by indenting fused silica which has a well characterized indentation response. Before each indentation, the tip was cleaned by indenting the polymer substrate in which the prosthesis was embedded. Displacement control mode was used during the analysis in order to prevent the large displacements typically observed during fracture under load control. The purpose of this approach is to enable the failure mode of the pillars to be examined in more detail through SEM imaging after fracture. Video records of the indentation were also collected to provide further insight into the pillar failure behavior as shown in Videos 1 and 2 (see supplementary information).
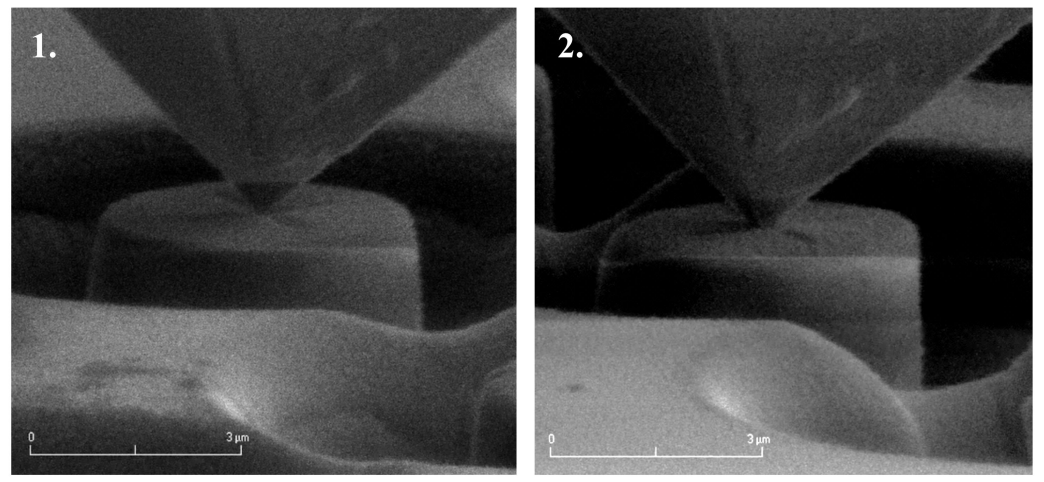

Video 1 and 2. Video snapshots of cube corner nanoindentation splitting in a) YPSZ ( $9 \mu \mathrm{m}$ from interface) and b) porcelain (11 $\mu \mathrm{m}$ from interface). The more ductile fracture of YPSZ and brittle response of porcelain are demonstrated in these two videos. http://dx.doi.org/10.1117/12.2199217.1 ; http://dx.doi.org/10.1117/12.2199217.2 
Preliminary micropillar indentation splitting was performed using the approach of Sebastiani et al. [27]. A diamond Berkovich three-sided pyramid with the total included angle of $142.3^{\circ}$ was used to perform this analysis. This allowed the use of the calibrated values of pre-multiplier $\left(\gamma_{B}\right)$ previously reported for this technique [27]. The relationship between the Berkovich-derived fracture toughness $\left(K_{B}\right)$, critical failure load $\left(P_{C}\right)$ and pillar radius $(R)$ is then given by:

$$
K_{B}=\gamma_{B} \frac{P_{C}}{R^{3 / 2}}
$$

The large included angle associated with this indenter type is known to minimise the sliding between the indenter tip and pillar surfaces. This ensures that the fracture load is less dependent upon the friction coefficient between these two surfaces (a parameter which is typically unknown). However, the shallow viewing angle imposed by the tip shape presents difficulties in aligning the indenter tip to the center of the pillar. The SEM imaging performed is constrained by the geometry of the indenter and thereby reduces the precision with which this can be performed along the direction of the beam. In total 9 indents were performed in the YPSZ using this approach and 3 indents were performed in porcelain, taking an average time of 40 minutes per point. All of these points were selected to be far from the interface where the variation in fracture toughness was expected to be small.

In order to improve the alignment between the indenter tip and the center of the pillar and reduce the time required to indent the remaining 48 pillars, the decision was made to use a diamond cube corner indenter tip. The reduced included angle of $90^{\circ}$ associated with this tip geometry increases the sensitivity of the technique to the friction between diamond and the substrate material, and means that the value of $\gamma_{B}$ previously published by Sebastiani et al. [27] is no longer valid. Despite this, direct comparison between the fracture toughness results obtained using Berkovich and cube corner indentation tips can be used to provide an estimate of the cube corner indentation load pre-multiplier $\left(\gamma_{C}\right)$ for a given material, as outlined in Section 3.2. Indentation was then performed on all of the remaining pillars at the average time of 11 minutes per point. Imaging of the fractured pillars was preformed using Hitachi S-4800 (CFE) SEM at Empa, Thun, Switzerland, with imaging direction aligned with the substrate surface normal.

\section{RESULTS}

\subsection{Young's modulus and hardness variation at the YPSZ-porcelain interface}

In order to perform fracture toughness evaluation through micropillar splitting, knowledge of the variation of Young's modulus $(E)$ and hardness $(H)$ within the region of interest is required. The ratio between these parameters can then be used to calculate the load pre-multiplier $\gamma_{B}$ through Finite Element (FE) simulations. A plot of the relationship between $E / H$ and $\gamma_{B}$ was recently published by Sebastiani et al [23], as shown in Figure 2.

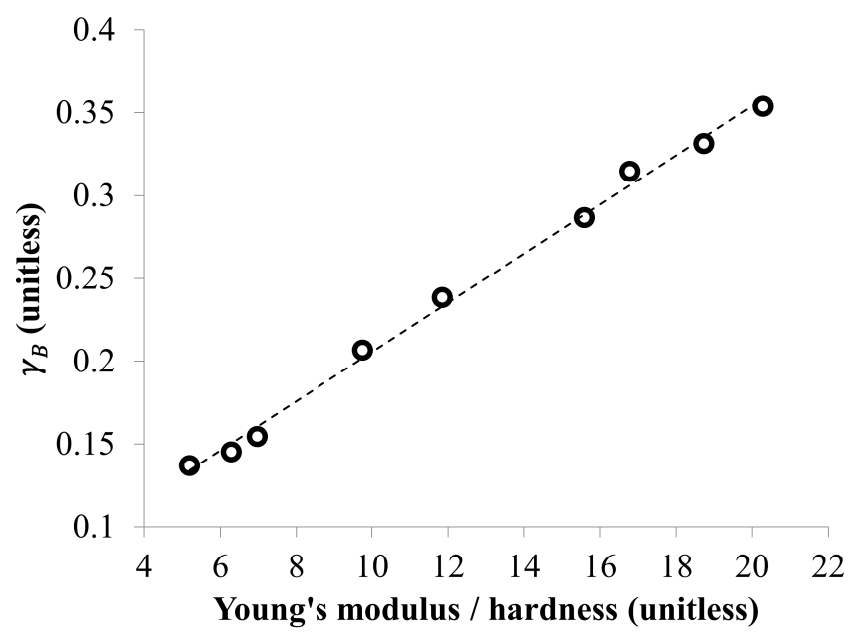

Figure 2. Plot of Berkovich load pre-multiplier $\left(\gamma_{B}\right)$ as a function of the ratio between Young's modulus and hardness $(E /$ $H)$ as published by Sebastiani et al. [23]. Linear least squares fitting has been applied to the dataset to obtain the expression given in Equation 2. 
Least squares fitting of Figure 2 can be used to demonstrate that for $E / H$ values ranging from 5 to 21 this relationship is approximately linear with the following relationship:

$$
\gamma_{B}=0.0149 \frac{E}{H}+0.057
$$

The spatial distribution of Young's modulus and yield strength (or fracture strength) across the interface of an identical YPSZ-porcelain dental prosthesis was recently published in a micropillar compression by Lunt et al. [11]. The quantitative values obtained using this approach are known to be independent of the high magnitude residual stresses in the near-interface region due to the full stress relaxation induced in the pillars prior to compression. Young's modulus values obtained from this analysis were close to the published values. For YPSZ this value was $216 \mathrm{GPa}$ compared to $210 \mathrm{GPa}$ provided by the manufacturer [28], and for the porcelain this estimate was $70 \mathrm{GPa}$ compared to the bulk value of $60 \mathrm{GPa}$ previously reported in the literature [30].

Nanoindentation hardness profiling has previously been successfully performed across the YPSZ-porcelain interface [15, 16]. However, the influence of residual stress and the onset of brittle fracture are known to influence hardness measurements obtained for the system [31]. To gain insight into the likely trends of hardness across the interface, the approximately constant relationship that exists between yield strength $\left(\sigma_{y}\right)$ and hardness for a given material can be exploited. Although the exact relationship between these two parameters is dependent upon the specific substrate, a good approximation for the relationship is that hardness equates three times the yield stress [32]. Comparison between the average yield stress obtained by micro-pillar compression [11] and those provided in the literature, demonstrate that this is a good approximation for YPSZ (13.5 GPa compared to $12.75 \mathrm{GPa}$ provided by the manufacturer [28]) but not for porcelain (10.3 GPa compared to $5.4 \mathrm{GPa}$ published in the literature [33]). These differences are likely associated with size effects and different microstructures of the two materials.

Therefore, in order to provide insight into the likely variation of the ratio between Young's modulus and hardness across the YPSZ - porcelain interface, the results obtained by micropillar compression [11] were used in the following approximation:

$$
\frac{E(x)}{H(x)} \approx \frac{E(x)}{3 \sigma_{y}(x)^{\prime}}
$$

where $x$ is the distance from the interface. The standard deviations of $E$ and $\sigma_{y}$ were used to calculated the $95 \%$ confidence intervals of $E / H$ and the results are shown in Figure 3.

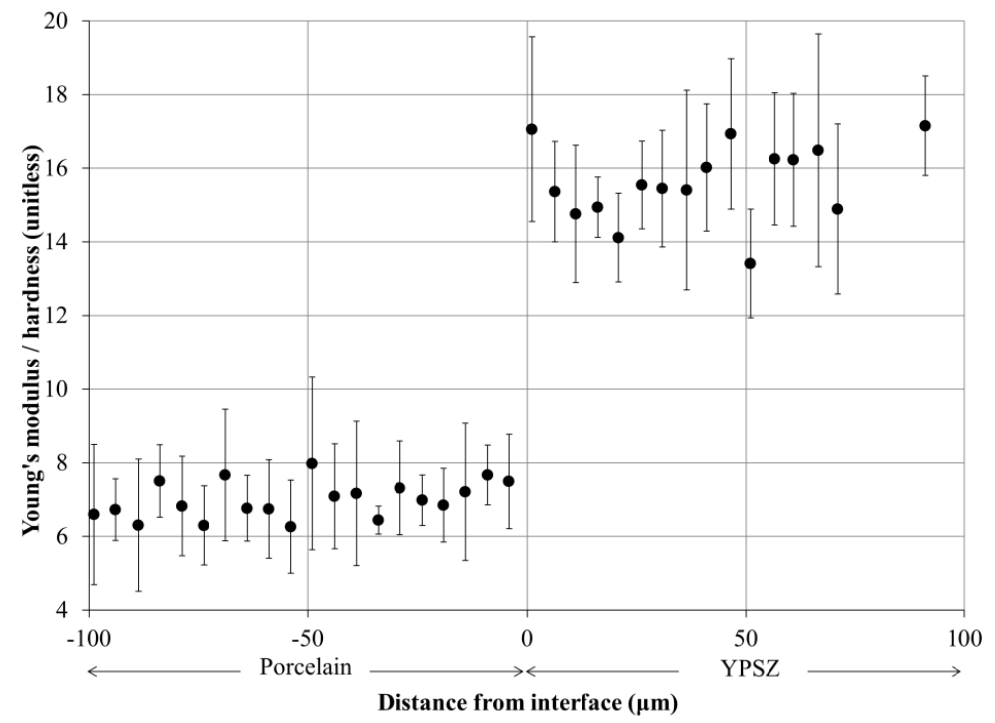

Figure 3. The variation of the Young's modulus to hardness ratio as a function of distance from the YPSZ-porcelain interface. The data values obtained for this distribution are taken from micropillar compression study published by Lunt et al. [11] and the use of the approximation that hardness approximately equals 3 times the yield stress The error bars indicate the $95 \%$ confidence intervals of each value. 
Critical examination of Figure 3 reveals that within each material the scatter of $E / H$ is generally equal to or smaller than the $95 \%$ confidence intervals of each value. This means that in terms of statistical significance, given the scatter of the results obtained experimentally, the value $E / H$ can be considered to be approximately constant in both YPSZ and porcelain. Further, as highlighted in Equation 2, it can be seen that moderate changes in the value of $E / H$ result in very small changes in $\gamma_{B}$, i.e. the fracture toughness estimate is robust with respect to minor deviations in $E / H$. This uniform $E / H$ approximation in both YPSZ and porcelain was used in the remainder of the analysis.

It was decided that the literature values of Young's modulus and hardness would be used to calculate the $E / H$ ratio for both YPSZ and porcelain (Table 1). Bulk estimates are typically more reliable than the results of micro-mechanical testing, and the use of these values is also consistent with the approach implemented by Sebastiani et al. [27].

Table 1. Mechanical properties and indentation load pre-multipliers for YPSZ and porcelain. Literature values of Young's Modulus $(E)$, hardness $(H)$ and fracture toughness $(K)$ are given with references, along with the calculated values of the Berkovich and cube corner indentation load pre-multipliers $\left(\gamma_{B}\right.$ and $\gamma_{C}$, respectively). The average fracture toughness estimate obtained from Berkovich indentation $\left(K_{B}\right)$ is included for comparison. The standard deviation of each measurement has been included in the table where values are known.

\begin{tabular}{|l|c|c|c|c|c|c|}
\hline Material & $\boldsymbol{E}(\mathbf{G P a})$ & $\boldsymbol{H}(\mathbf{G P a})$ & $\boldsymbol{K}\left(\mathbf{M P a m}^{\mathbf{0 . 5}}\right)$ & $\boldsymbol{\gamma}_{\boldsymbol{B}}$ & $\boldsymbol{\gamma}_{\boldsymbol{C}}$ & $\boldsymbol{K}_{\boldsymbol{B}}(\mathbf{M P a m}$ \\
\hline YPSZ & $210[28]$ & $12.75 \pm 1.96[28]$ & $5.00[28]$ & $0.31 \pm 0.02$ & $1.10 \pm 0.05$ & $4.79 \pm 0.24$ \\
\hline Porcelain & $60[34]$ & $5.40 \pm 0.20[33]$ & $2.75 \pm 0.25[33]$ & $0.22 \pm 0.01$ & $0.41 \pm 0.04$ & $2.65 \pm 0.27$ \\
\hline
\end{tabular}

\subsection{Micropillar indentation splitting}

After successfully fracturing each of the 60 FIB milled pillars, analysis was performed on the load-displacement curves obtained from the analysis. Distinct differences were observed between the fracture of the YPSZ and porcelain micropillars, examples of which are shown in Figure 4. In the case of porcelain, brittle failure occurred without the onset of plasticity. However in the case of YPSZ, a gradual decrease in the load-displacement plot was observed prior to failure. Despite these differences, consistent estimates of the critical load for instability $\left(P_{C}\right)$ were obtained from each of the pillars corresponding to the point where large drops were observed in the load.

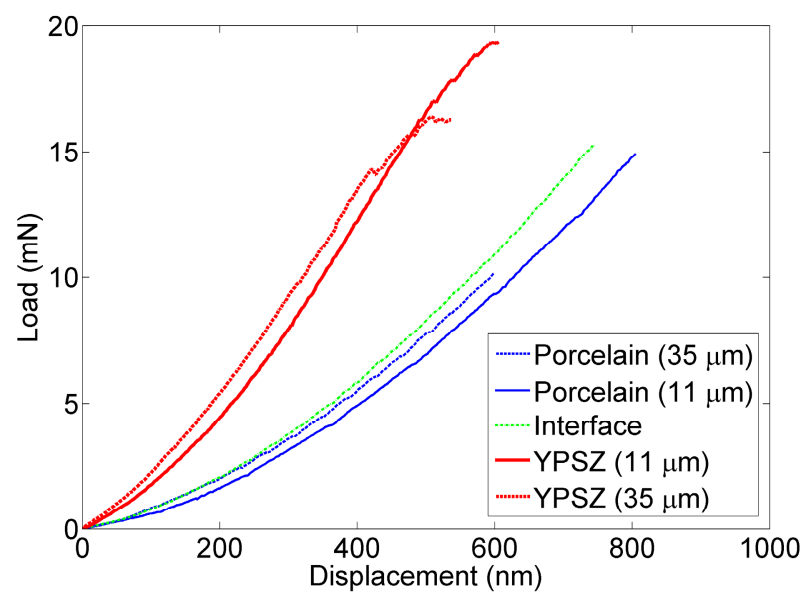

Figure 4. Typical load-displacement plots for YPSZ, porcelain and the interface up to the point of brittle failure. The distance from the interface to the pillar center is given in the plot legend. YPSZ shows plasticity prior to critical failure whereas no reduction in gradient is observed in porcelain or the interface indentation.

In order to obtain estimates of the fracture toughness of each of the pillars, the values of Berkovich indentation load premultiplier $\gamma_{B}$ for each material were obtained. These were based on the bulk values of Young's modulus and hardness for YPSZ and porcelain given in Table 1 and Equation 2 [23]. Estimates of Berkovich fracture toughness were then obtained using Equation 1. In order to provide comparisons with the cube corner indentations, the average value of $K_{B}$ was determined at positions at which both techniques had been applied; 60,70 and 80 $\mu \mathrm{m}$ from the interface for porcelain and 35, 50 and $60 \mu \mathrm{m}$ from the interface for YPSZ. The averages and standard deviations of $K_{B}$ obtained from 
these three points are given in Table 1. These estimates were then treated as absolute measures of fracture toughness in order to optimize $\gamma_{C}$, the cube corner indentation load pre-multiplier for each material. This parameter relates the cube corner fracture toughness $K_{C}$ to the critical instability load and pillar radius $R$, through the expression:

$$
K_{C}=\gamma_{C} \frac{P_{C}}{R^{3 / 2}}
$$

The magnitude and standard deviation of $\gamma_{C}$ for YPSZ and porcelain obtained from this analysis are given in Table 1. These estimates were then used in combination with the critical fracture toughness values to obtain estimates of the $K_{C}$ in the YPSZ and porcelain near-interface regions. At the locations where both YPSZ and porcelain were contained within the pillar, an average value of $\gamma_{C}=0.5 \times\left(\gamma_{C}^{Z i r}+\gamma_{C}^{P o r}\right)$ was used to determine $K_{C}$. The standard deviations of the fracture toughness values were obtained from the combined uncertainties of each of the parameters used in their calculation. The results of this analysis are shown in Figure 5.

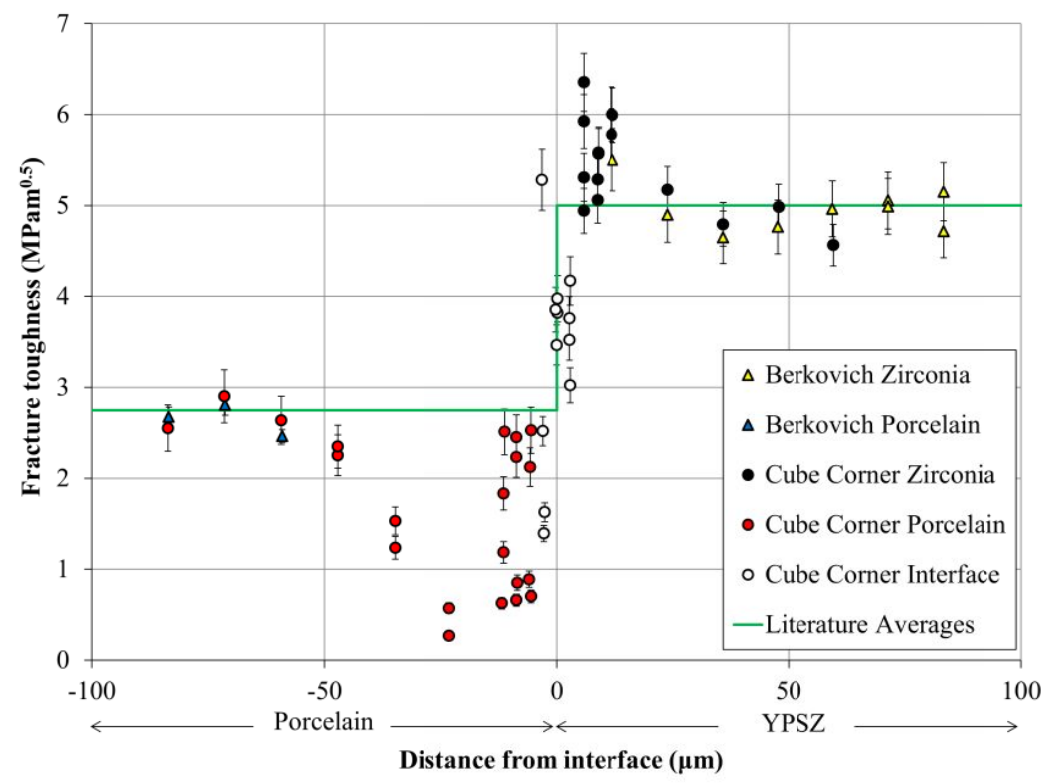

Figure 5. Fracture toughness variation across the YPSZ - porcelain interface showing YPSZ on the right (positive distances) and porcelain on the left (negative distances). The results from both Berkovich and cube corner indentation are shown along with the average values obtained from the literature [28, 33]. At the interface an average of the $\gamma$ values for the two materials was used to calculate the fracture toughness. The error bars shown indicate the $95 \%$ confidence intervals of each measurement.

Examination of the SEM images of the fractured pillars revealed that the average offset between the center of the pillar and the indenter tip was less than $100 \mathrm{~nm}$ for the cube corner indentations, compared with $100-300 \mathrm{~nm}$ for the Berkovich tip. Four main failure types were observed in the pillars depending upon the substrate material, location and indenter tip geometry as shown in Figure 6. Examination of the porcelain micropillars revealed that for many of the tested positions, the indentation had been sufficient to fully fracture the stub, leaving only the base or a small section of the pillar (Figure 6a). The smooth, sharp facets observed on the remaining surfaces suggested that porcelain failure was brittle, in contrast to the gradual failure observed in YPSZ. In near-interface pillars, which contained both YPSZ and porcelain within a single pillar (Figure 6b), a different failure type was widely observed. Typically the pillar was found to fracture into two pieces, with the direction of fracture being closely aligned to the interface direction. The fracture observed in these pillars was sharp suggesting a single failure event, and the crack was typically induced within the porcelain at a distance of between $1-2 \mu \mathrm{m}$ from the interface. Cube corner indentation of YPSZ resulted in pillar splitting along the sharp edges of the indent as shown in Figure 6c. Fracture surfaces were highly faceted suggesting failure may not have occurred fully in a single fracture event, but increased gradually during continued application of load. In contrast to this behavior under cube corner tip, Berkovich indentation cracks were localized to pillar edges in YPSZ, this was believed to be associated sub-surface bulk cracking which propagated to the pillar surface as shown in Figure 6d. 

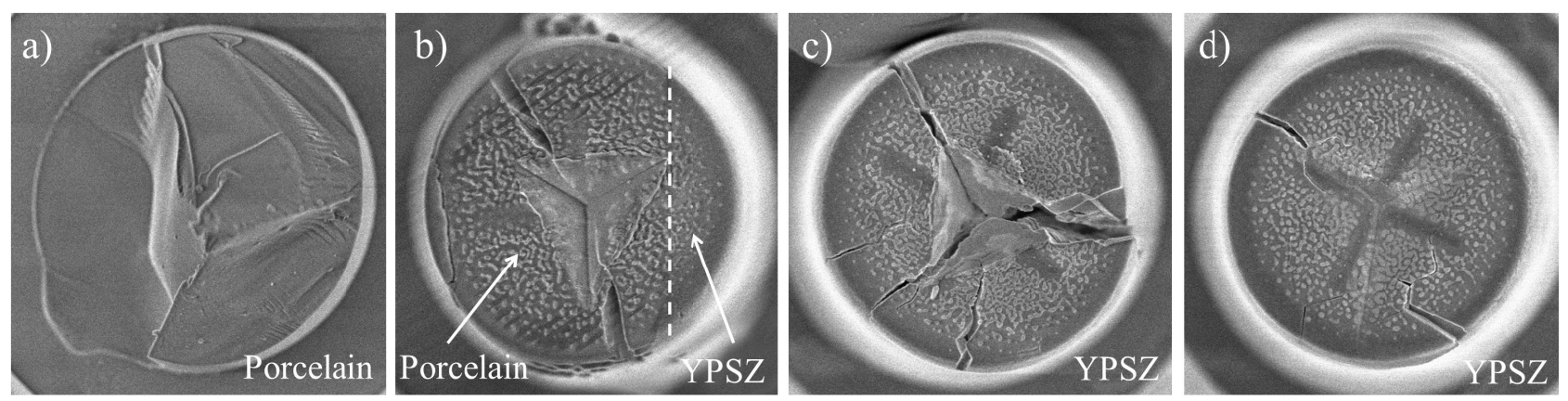

Figure 6. SEM images of fractured pillars. a) Complete brittle failure of the porcelain micropillar located $47 \mu \mathrm{m}$ from the interface. The remaining stub base is shown, along with the smooth fracture surfaces. b) Typical near-interface cube corner micropillar failure showing the pillar splitting into two halves. Examination of multiple interface fractures revealed that the fracture direction was often closely aligned to the interface. c) Typical YPSZ cube corner tip fracture showing multiple cracks from the center to the edge of a pillar located $11 \mu \mathrm{m}$ from the interface. $d)$ Berkovich tip indentation of the YPSZ micropillar located at $35 \mu \mathrm{m}$ from the interface. Two cracks can be observed from the edge of the pillar running towards the center.

\section{DISCUSSION}

Examination of the fracture toughness variation across the interface in Figure 5 reveals that beyond $50 \mu \mathrm{m}$ from the interface in porcelain and beyond $25 \mu \mathrm{m}$ in YPSZ, the fracture toughness is approximately constant and equal to the average bulk values published in the literature $[28,33]$. This confirms that the results obtained though the of Berkovich pillar splitting approach developed by Sebastiani et al $[23,27]$ are reliable in both YPSZ and porcelain. Further, the good agreement in numerical fracture toughness confirms that the calibration of $\gamma_{C}$ outlined in Section 3.2 is valid.

The values of $\gamma_{C}$ for YPSZ and porcelain were determined to be 1.10 and 0.41 respectively. Pillar splitting using a cube corner indentation tip has not previously been reported in the literature, and therefore no comparative results are available. The magnitudes of these pre-multipliers are several times larger than the equivalent $\gamma_{B}$ values for each material. This is known to be associated with the sharper indentation and increased surface sliding associated with a cube corner indent, which induces a different type of pillar failure as shown in Figures 5a and 5b, but also increases the sensitivity of the technique to the friction coefficient between the diamond tip and the substrate material. Despite these differences, the variation in fracture toughness obtained through cube corner indentation appears to be realistic and can begin to explain some of the failures observed in this system.

In order to gain insight into the scatter observed within the very near interface region $(<15 \mu \mathrm{m})$ a systematic examination was performed to assess factors which may have influenced these values. No correlation could be observed between the fracture toughness and the misalignment between the pillar center and the indentation position. This study therefore reveals that the pillar splitting approach is robust to minor errors in indenter positioning. Analysis was also performed to assess the impact of spatial positioning (along the interface direction), pillar diameter and pillar height on the fracture toughness values obtained. No trends could be identified in any of these parameters, suggesting that the scatter observed in the near-interface region is instead more likely to be representative of the random fracture toughness variation.

Distinct differences were observed between the failure modes in YPSZ, porcelain and the interface containing both YPSZ and porcelain. Video 1 and Figure 4 both demonstrate that the failure of YPSZ is gradual and occurs by crack propagation and slow growth under increasing load. This results in a reduction in the gradient on the load-displacement curve which is indicative of hardening or softening. The multi-faceted crack surfaces observed in the fractured pillars (Figure 6c) provide further evidence that YPSZ failure is associated with gradual crack growth. In contrast to this behavior, Video 2 and Figure 4 highlight the brittle failure induced in porcelain. There is no change in gradient on the load-displacement curve and complete pillar fracture occurs at a point at which there were no visible cracks in the pillar surface.

In the near interface region, a distinct failure type is observed which is believed to be a combination of both the YPSZ and porcelain failure mechanisms, i.e. preferential pillar splitting along the direction of the YPSZ-porcelain interface. Planar interfaces are implicitly associated with orientational dependence and therefore it is perhaps unsurprising that the 
half pillar splitting observed in the interface region is preferentially aligned to the interface. What is however more surprising is that failure was not observed at the interface location, where a step change in mechanical properties is expected, or at the pillar center. Failure was found to be more likely within the near-interface porcelain at a distance of between $1-2 \mu \mathrm{m}$ from the interface. This length scale correlates well with the position of nanoscale voiding observed in porcelain by Lunt et al. [11] $(0.4-1.5 \mu \mathrm{m}$ from the interface) suggesting that there may be an underlying structural weakness in the porcelain at this location. The random nature of void type features may also explain the large scatter observed in the fracture toughness of the near-interface porcelain pillars.

Clear differences can be observed between the literature average values of fracture toughness and those obtained micropillar splitting for both YPSZ and porcelain in the near-interface region (Figure 5). A clear reduction in fracture toughness (by up to $\sim 90 \%$ ) is observed in porcelain starting approximately $50 \mu \mathrm{m}$ from the interface and extending to the near interface location. This variation is highly consistent in the pillars between 50 and $15 \mu \mathrm{m}$ from the interface, suggesting that this weakness may be associated with a gradual variation in mechanical properties caused, for example, by elemental diffusion or long-range grain refinement effects, both of which are known to influence the fracture toughness of porcelain [35]. In contrast to this behavior, at distances less than $15 \mu \mathrm{m}$ from the interface, a large amount of scatter is observed within the data suggesting more statistically driven weaknesses than those observed at larger distances. The fracture toughness estimates within this range vary from $0.6-2.5 \mathrm{MPam}^{0.5}$ which demonstrates that the bulk mechanical behavior is present within some near-interface positions.

The variation of fracture toughness within the interface pillars (containing both YPSZ and porcelain) demonstrates relatively high levels of scatter with an average value of $3.8 \mathrm{MPam}^{0.5}$. This corresponds well with the average literature fracture toughness values of YPSZ and porcelain of $3.9 \mathrm{MPam}^{0.5}$. Despite this consistency, the fracture toughness in pillars containing mostly porcelain shows high levels of scatter. Of the four pillars measured, three are within the scatter observed in the neighboring porcelain however one high toughness pillar $\left(5.3 \mathrm{MPam}^{0.5}\right)$ was observed at this location, this may be an outlier or perhaps is more representative of failure within YPSZ.

In YPSZ the fracture toughness is approximately constant and equal to the literature value of $5 \mathrm{MPam}^{0.5}$ up a distance of $15 \mu \mathrm{m}$ from the interface. In the near-interface region, an increase in fracture toughness is observed up to an average value of $5.5 \mathrm{MPam}^{0.5}$. An increase in scatter is also observed in this region with values between $4.9-6.4 \mathrm{MPam}^{0.5}$ being observed. This again suggests that this behavioral change is statistically driven, rather than a gradual transition and therefore is likely to be associated with local features. At present the exact origin of this increased toughness is not clear. One explanation may be high elemental composition sensitivity of YPSZ fracture toughness $[18,36]$ and the variations in the concentration of $\mathrm{Si}, \mathrm{K}, \mathrm{Na}$ and $\mathrm{Al}$ known to be present within the first $5 \mu \mathrm{m}$ of the interface [11]. Perhaps more likely, given the scatter in the fracture toughness values obtained, is the grain size dependence of YPSZ fracture toughness [37]. Larger grains of YPSZ are more likely to transform from a tetragonal to a monoclinic phase during stress application, increasing the amount of energy absorbed by the material during fracture. TEM of the YPSZ-porcelain interface has previously highlighted YPSZ grain size variations at this location, including the presence of relatively large grains $\left(1-2 \mu \mathrm{m}^{2}\right)$ within the first $10 \mu \mathrm{m}$ of the interface [11]. The scatter observed may therefore be dependent upon the presence, or lack of, a large grain within the gauge volume under consideration.

\section{CONCLUSIONS}

This study is the first published work which outlined the application of spatially resolved fracture toughness using nanoindentation and pillar splitting. The approach has been shown to provide reliable estimates of fracture toughness in the ceramics YPSZ and porcelain and has been used to investigate the variation of fracture toughness within a sample of commercial interest; the YPSZ-porcelain interface in dental prostheses. Further, cube corner indentation (which can be aligned more quickly and precisely to pillar centers) has been shown to provide realistic estimates of fracture toughness by making use of the critical failure load pre-multiplier $\gamma_{C}$. Estimates of $\gamma_{C}$ for both YPSZ and porcelain were obtained by comparison with the Berkovich indentation approach previously published by Sebastiani et al. [23, 27].

Near interface YPSZ has been shown to fail through a gradual, 'plastic' type fracture and has shown to be consistent with literature estimates of fracture toughness at distances beyond $15 \mu \mathrm{m}$ from the interface. In the very near interface region, a 10\% increase in fracture toughness was observed, along with an increase in scatter. This may be associated with grain size variation and the associated impact on fracture toughness in YPSZ. 
In pillars which contained both YPSZ and porcelain, a distinctive half pillar splitting type failure was observed which was typically aligned with the interface and was positioned in the porcelain at a distance of $1-2 \mu \mathrm{m}$ from the interface. This behavior is believed to be associated with the nanoscale voiding previously observed in porcelain at this location.

Porcelain micropillar failure was found to be brittle in nature, with no plasticity or cracking observed before complete failure of the micropillar. Scatter was observed within the very near-interface $(<15 \mu \mathrm{m})$ fracture toughness values suggesting a statistically driven failure which was dependent upon local features. Further variation in fracture toughness was observed up to $50 \mu \mathrm{m}$ from the interface which demonstrated a gradual and consistent reduction in toughness (of up to $\sim 90 \%$ ). This large reduction in fracture toughness, in combination with the high magnitude residual stresses at this location, has the potential to explain the high failure rates observed in this micromechanical system.

The insights gained by this study can be used in combination with other recent high resolution mechanical studies of the YPSZ-porcelain interface in order to develop improved models of the evolution of residual stress, microstructure and mechanical behavior. These simulations can be used to optimize the thermal processing routes applied during manufacture in order to reduce the impact of localized residual stress and fracture toughness variation, with the ultimate goal of reducing prosthesis failure rates.

\section{ACKNOWLEDGMENTS}

The European Project EU FP7 project iStress (604646) is acknowledged for the funding support provided to the MultiBeam Laboratory for Engineering Microscopy (MBLEM), where the FIB milling of the micropillars was carried out. Alexander Korsunsky wishes to acknowledge the support of the Research Complex at Harwell for the Centre for In situ Processing Studies (CIPS). Gaurav Mohanty would like to acknowledge funding from Empa Postdoc program co-funded by FP7: Marie Curie Actions.

\section{REFERENCES}

[1] I. Denry, and J. R. Kelly, "State of the art of zirconia for dental applications," Dental Materials, 24(3), 299-307 (2008).

[2] B. Basu, "Toughening of yttria-stabilised tetragonal zirconia ceramics," International Materials Reviews, 50(4), 239-256 (2005).

[3] R. Hmaidouch, and P. Weigl, "Tooth wear against ceramic crowns in posterior region: a systematic literature review," International journal of oral science, 5(4), 183-190 (2013).

[4] N. R. F. A. Silva, E. A. Bonfante, R. A. Zavanelli et al., "Reliability of Metalloceramic and Zirconia-based Ceramic Crowns," Journal of dental research, 89(10), 1051-1056 (2010).

[5] M. Baldassarri, C. F. J. Stappert, M. S. Wolff et al., "Residual stresses in porcelain-veneered zirconia prostheses," Dental Materials, 28(8), 873-879 (2012).

[6] R. Belli, R. Frankenberger, A. Appelt et al., "Thermal-induced residual stresses affect the lifetime of zirconiaveneer crowns," Dental Materials, 29(2), 181-190 (2013).

[7] B. Al-Amleh, J. N. Waddell, K. Lyons et al., "Influence of veneering porcelain thickness and cooling rate on residual stresses in zirconia molar crowns," Dental Materials, 30(3), 271-280 (2014).

[8] A. J. G. Lunt, S. Kabra, J. Kelleher et al., "Tensile Secondary Creep Rate Analysis of a Dental Veneering Porcelain,” Thin Solid Films, In Press - Corrected Proof, (2015).

[9] A. J. G. Lunt, T. K. Neo, and A. M. Korsunsky, "An Electron Microscopy Study of Sintering in Three Dental Porcelains," Proc WCE, 2, (2015).

[10] D. Liu, J. P. Matinlinna, J. K.-H. Tsoi et al., "A new modified laser pretreatment for porcelain zirconia bonding,” Dental Materials, 29(5), 559-565 (2013).

[11] A. J. G. Lunt, G. Mohanty, S. Ying et al., "A Comparative Transmission Electron Microscopy, Energy Dispersive X-ray Spectroscopy and Spatially Resolved Micropillar Compression Study of the Yttria Partially Stabilised Zirconia-Porcelain Interface in Dental Prosthesis," Thin Solid Films, In Press - Corrected Proof, (2015).

[12] Y. Kawai, M. Uo, and F. Watari, "Microstructure evaluation of the interface between dental zirconia ceramics and veneering porcelain," Nano Biomedicine, 2(1), 31-36 (2010).

[13] A. Grigore, S. Spallek, A. Petschelt et al., "Microstructure of veneered zirconia after surface treatments: A TEM study,” Dental Materials, 29(11), 1098-1107 (2013). 
[14] T. F. Alghazzawi, and G. M. Janowski, "Evaluation of zirconia-porcelain interface using X-ray diffraction," International journal of oral science, 7(3), 187-195 (2015).

[15] M. Sebastiani, F. Massimi, G. Merlati et al., "Residual micro-stress distributions in heat-pressed ceramic on zirconia and porcelain-fused to metal systems: Analysis by FIB-DIC ring-core method and correlation with fracture toughness," Dental Materials, (2015).

[16] Y. Zhang, M. Allahkarami, and J. Hanan, "Measuring residual stress in ceramic zirconia-porcelain dental crowns by nanoindentation," J Mech Behav Biomed Mater, 6, 120-127 (2012).

[17] A. Selçuk, and A. Atkinson, "Strength and Toughness of Tape-Cast Yttria-Stabilized Zirconia," Journal of the American Ceramic Society, 83(8), 2029-2035 (2000).

[18] K. Tsukuma, K. Ueda, and M. Shimada, "Strength and Fracture Toughness of Isostatically Hot-Pressed Composites of A12O3 and Y2O3-Partially-Stabilized ZrO2," Journal of the American Ceramic Society, 68(1), C-4-C-5 (1985).

[19] R. Morena, P. Lockwood, and C. Fairhurst, "Fracture toughness of commercial dental porcelains," Dental Materials, 2(2), 58-62 (1986).

[20] S. Scherrer, J. R. Kelly, G. D. Quinn et al., "Fracture toughness (K IC) of a dental porcelain determined by fractographic analysis," Dental Materials, 15(5), 342-348 (1999).

[21] M. Guazzato, K. Proos, L. Quach et al., "Strength, reliability and mode of fracture of bilayered porcelain/zirconia (Y-TZP) dental ceramics," Biomaterials, 25(20), 5045-5052 (2004).

[22] A. G. Evans, and E. A. Charles, "Fracture toughness determinations by indentation," Journal of the American Ceramic Society, 59(7-8), 371-372 (1976).

[23] M. Sebastiani, K. Johanns, E. Herbert et al., "Measurement of fracture toughness by nanoindentation methods: Recent advances and future challenges," Current Opinion in Solid State and Materials Science, (2015).

[24] D. Di Maio, and S. Roberts, "Measuring fracture toughness of coatings using focused-ion-beam-machined microbeams," J Mater Res, 20(02), 299-302 (2005).

[25] A. Riedl, R. Daniel, M. Stefenelli et al., "A novel approach for determining fracture toughness of hard coatings on the micrometer scale," Scripta Materialia, 67(7-8), 708-711 (2012).

[26] S. Liu, J. M. Wheeler, P. R. Howie et al., "Measuring the fracture resistance of hard coatings," Applied Physics Letters, 102(17), 171907 (2013).

[27] M. Sebastiani, K. Johanns, E. Herbert et al., "A novel pillar indentation splitting test for measuring fracture toughness of thin ceramic coatings," Philosophical Magazine((ahead of print)), 1-17 (2014).

[28] "Zenotec $\mathrm{Zr} \quad$ Bridge," http://www.wieland-dental.de/en/products/zenotec/materials/zenotec-zr-bridge/ $(10 / 09 / 15)$.

[29] “IPS e.max Ceram,” http://www.ivoclarvivadent.co.uk/en/products/all-ceramics/ips-emax-technicians/ipsemax-ceram (19/02/15).

[30] M. Uchic, P. Shade, and D. Dimiduk, "Micro-compression testing of fcc metals: A selected overview of experiments and simulations," JOM-J Min Met Mat S, 61(3), 36-41 (2009).

[31] W. C. Oliver, and G. M. Pharr, "An improved technique for determining hardness and elastic modulus using load and displacement sensing indentation experiments," Journal of Materials Research, 7(06), 1564-1583 (1992).

[32] P. Zhang, S. X. Li, and Z. F. Zhang, "General relationship between strength and hardness," Materials Science and Engineering: A, 529, 62-73 (2011).

[33] "IPS e.max ${ }^{\circledR}$ Ceram Scientific Documentation," www.roedentallab.com/downloads/emaxceramicdata.pdf $(19 / 02 / 15)$.

[34] M. D. Uchic, and D. M. Dimiduk, "A methodology to investigate size scale effects in crystalline plasticity using uniaxial compression testing," Mater Sci Eng A, 400(0), 268-278 (2005).

[35] J. Quinn, V. Sundar, and I. Lloyd, "Influence of microstructure and chemistry on the fracture toughness of dental ceramics," Dental Materials, 19(7), 603-611 (2003).

[36] O. Vasylkiv, Y. Sakka, and V. V. Skorokhod, "Hardness and fracture toughness of alumina-doped tetragonal zirconia with different yttria contents," Materials Transactions, 44(10), 2235-2238 (2003).

[37] M. Trunec, and Z. Chlup, "Higher fracture toughness of tetragonal zirconia ceramics through nanocrystalline structure," Scripta Materialia, 61(1), 56-59 (2009). 